\title{
Effects of chlorpheniramine on emergence agitation after general anesthesia for ureteroscopic stone surgery: a retrospective cohort study
}

\section{Choon-Kyu Cho, Minhye Chang, Seok-Jin Lee, Sung-Ae Cho, and Tae-Yun Sung}

Department of Anesthesiology and Pain Medicine, Konyang University Hospital, Konyang University College of Medicine, Daejeon, Korea
Received June 22, 2021

Revised August 4, 2021

Accepted August 4, 2021

\section{Corresponding author}

Tae-Yun Sung, M.D., Ph.D.

Department of Anesthesiology and Pain Medicine, Konyang University Hospital, Konyang University College of Medicine, 158 Gwanjeodong-ro, Seo-gu, Daejeon 35365, Korea

Tel: 82-42-600-9316

Fax: 82-42-545-2132

E-mail: unt1231@naver.com
Background: The presence of a urinary catheter, postoperative pain, and postoperative nausea and vomiting are risk factors for emergence agitation (EA). Antimuscarinic agents are primary agents used in the prevention and treatment of urinary catheter-related bladder discomfort. Chlorpheniramine has antimuscarinic, antinociceptive, and antiemetic effects. This retrospective study investigated the role of chlorpheniramine in EA prevention following ureteroscopic stone surgery.

Methods: Of 110 adult patients who underwent ureteroscopic stone surgery under general anesthesia between January and December 2019, the medical records of 93 patients were analyzed retrospectively. The patients were divided into control $(n=52)$ and chlorpheniramine $(n=41)$ groups according to the receipt of intravenous chlorpheniramine before the induction of anesthesia. The incidence and severity of EA were compared between the groups as primary and secondary endpoints, respectively. The effects of chlorpheniramine on the requirement for inhalation anesthetic (desflurane) during surgery, changes in mean blood pressure and heart rate during emergence, and adverse events were also compared.

Results: The incidence (21.2\% in the control group, $24.4 \%$ in the chlorpheniramine group) and severity of EA did not differ between groups. The intraoperative requirement for desflurane, changes in mean blood pressure and heart rate during emergence, and adverse events were also similar between groups.

Conclusions: Chlorpheniramine was not associated with a decrease in EA incidence or severity in patients who underwent ureteroscopic stone surgery.

Keywords: Anesthesia; Chlorpheniramine; Emergence agitation; Incidence; Urinary catheter. 
urinary catheters, compared to $9.8-13.6 \%$ in patients undergoing urological surgery $[3,4]$. Presence of a urinary catheter is a known risk factor for EA [1-4]. Therefore, antimuscarinic agents are often used to prevent and treat catheter-related bladder discomfort (CRBD) [5].

Chlorpheniramine is an alkylamine first-generation potent $\mathrm{H}_{1}$ antihistamine generally used to prevent and treat hypersensitivity and allergic disorders [6,7], but also has sedative, local anesthetic, and antimuscarinic effects [6-8]. In addition, chlorpheniramine has an antiemetic effect and can be used to prevent and treat postoperative nausea and vomiting (PONV) [9]. These effects of chlorpheniramine are expected to attenuate EA by reducing PONV, postoperative pain, and CRBD [1-4], but at the same time, anticholinergic use is also a risk factor for EA [10]. Therefore, the effects of chlorpheniramine on EA are difficult to predict. Furthermore, no studies have evaluated the effect of chlorpheniramine in patients undergoing urological surgery and requiring urinary catheterization, with a high risk of EA. Thus, we aimed to evaluate the association between a single bolus dose ( $8 \mathrm{mg}$ ) of chlorpheniramine administered before the induction of anesthesia and EA in patients undergoing ureteroscopic stone surgery.

\section{MATERIALS AND METHODS}

This retrospective cohort study was approved by the Institutional Review Board (no. KYUH2020-01-005), and was registered with the Korea Clinical Research Information Service (http://cris.nih.go.kr) (no. KCT0004879). This study adhered to the STROBE checklist (https://www.strobe-statement.org/checklists/). Written informed consent was not obtained from patients due to the retrospective nature of the study. The medical records of patients who underwent elective ureteroscopic stone surgery under general anesthesia in our hospital between January 2019 and December 2019 were reviewed retrospectively. Two anesthesiologists were in charge of anesthesia for the urological surgeries; they used the same anesthetic agents and patient monitoring and extubation criteria, according to our institutional protocols. However, only one anesthesiologist intravenously administered 8 mg chlorpheniramine (Pheniramine inj ${ }^{\circledR}$, Yuhan Co., Korea) 5-10 min before the induction of anesthesia, in the absence of contraindication, to provide a sedative effect before anesthesia induction, to reduce PONV, and to help prevent perioperative hypersensitivity reactions $[11,12]$. According to this procedural difference, the patients were di- vided into chlorpheniramine and control groups. The inclusion criteria for this study were age 19-65 years and American Society of Anesthesiologists physical status classification I-III. All patients underwent elective ureteroscopic stone surgery under general anesthesia. The exclusion criteria were: the presence of a urinary catheter before anesthesia induction, induction of general anesthesia using a supraglottic airway device, cognitive or neuropsychological disorder, combined operation, contraindication to chlorpheniramine (e.g., prostatic hyperplasia, irritable bladder symptoms, bladder outlet obstruction, or glaucoma), and concomitant administration of steroids (e.g., dexamethasone or hydrocortisone) to prevent or treat an allergic reaction or anaphylaxis.

All patients were fasted for at least $8 \mathrm{~h}$ and arrived in the operating room without premedication. Patients in the chlorpheniramine group received intravenous $8 \mathrm{mg}$ chlorpheniramine 5-10 min before the induction of anesthesia, whereas patients in the control group did not. All subsequent anesthesia care and surgical procedures were the same in the two groups. Routine monitoring included electrocardiography, noninvasive blood pressure measurement, pulse oximetry, end-tidal carbon dioxide $\left(\mathrm{EtCO}_{2}\right)$ measurement, Patient State Index (PSI) (SedLine ${ }^{\circledR}$, Masimo Corp., USA) determination, and neuromuscular train-of-four (TOF) stimulation by acceleromyography (TOF-Watch $\mathrm{SX}^{\circledR}$, Organon Ltd., Ireland) on the adductor pollicis muscle. Anesthesia was induced with intravenous propofol (1.5-2 mg/ $\mathrm{kg}$ ) and fentanyl (1-2 $\mu \mathrm{g} / \mathrm{kg})$.

Endotracheal intubation was facilitated by rocuronium $(0.6 \mathrm{mg} / \mathrm{kg})$. Volume-controlled mechanical ventilation was initiated at a tidal volume of $8 \mathrm{ml} / \mathrm{kg}$ and a respiratory rate of 12 breaths/min. During the maintenance of anesthesia, the $\mathrm{EtCO}_{2}$ was maintained at 30-40 $\mathrm{mmHg}$ by adjusting the respiratory rate. Anesthesia was maintained with an oxygen/ nitrous oxide mixture (50:50) and 3-8 vol\% of the end-tidal concentration of desflurane to maintain the PSI at 25-50. All operations were performed in a lithotomy position. After surgery, each patient was catheterized with a Foley catheter, and the balloon was inflated with $5 \mathrm{ml}$ normal saline by the urologist. After urinary catheterization, the patient was moved to the supine position. Desflurane and nitrous oxide were stopped, and manual ventilation was performed with $100 \%$ oxygen at $6 \mathrm{~L} / \mathrm{min}$. The neuromuscular block was reversed with $50 \mu \mathrm{g} / \mathrm{kg}$ neostigmine and $10 \mu \mathrm{g} / \mathrm{kg}$ glycopyrrolate. The extubation criteria were: PSI $>75$, tidal volume $\geq 5$ $\mathrm{ml} / \mathrm{kg}$, spontaneous respiratory breathing rate $10-25 / \mathrm{min}$, 
TOF ratio $\geq 0.9$, and response to verbal commands. All patients were transferred to the post-anesthesia care unit (PACU) 5 min after extubation.

\section{Measurements}

Emergence was defined as the time interval between the discontinuation of all anesthetics (desflurane and nitrous oxide) and 5 min after extubation. The attending anesthetist (nurse), who has assessed agitation during emergence in all patients in our hospital since 2017, recorded the results on the patients' electronic medical charts [13]. EA was assessed using the Ricker Sedation-Agitation Scale (RSAS, 7 points; 1 = unarousable, 2 = very sedated, 3 = sedated, $4=$ calm and cooperative, 5 = agitated but responding calmly to verbal instructions, 6 = very agitated requiring restraint, 7 = pulling at the tracheal tube, trying to remove catheter or striking the staff) [14], and the highest RSAS score during emergence was recorded. RSAS scores $>5$ were considered to reflect EA and were included in the EA incidence. RSAS was used to classify EA according to severity. The incidence of EA was analyzed as the primary endpoint, and the severity of EA was analyzed as the secondary endpoint. We also analyzed the time to extubation, defined as the time between turning off the inhalation anesthetics and extubation.

Data on hemodynamic parameters (mean blood pressure and heart rate) before the induction of anesthesia, at the end of surgery, at extubation, and 5 min after extubation were collected and analyzed. In addition, the highest and lowest concentrations of desflurane administered during the maintenance of anesthesia were determined and compared to exclude the effect of a difference in the concentration of inhalation anesthetic on EA.

Among the PACU data, the severity of postoperative pain (evaluated using an 11-point numerical rating scale [NRS; 0 = no pain, 10 = worst pain imaginable]), requirements for analgesics and antiemetics, and all adverse events were analyzed.

\section{Statistical analyses}

The primary endpoint of this study was the incidence of EA. In a previous study [3], the incidence of EA was $63.5 \%$ in patients who received urinary catheters. Assuming that a $50 \%$ reduction in the incidence of EA after administration of chlorpheniramine would be clinically relevant, a sample of 38 patients per group was required, with a power of 0.8 and a two-sided a value of 0.05 .

The statistical analyses were performed using SPSS Statistics software (ver. 18.0 for Windows, IBM Corp., USA). Continuous variables were analyzed using Student's $t$-test or the Mann-Whitney $U$ test, depending on the KolmogorovSmirnov normality test result. Categorical variables were analyzed with the $\chi^{2}$ test, the $\chi^{2}$ test for trends (linear-by-linear association), or Fisher's exact test, as appropriate. After obtaining the results of normality and Mauchly's sphericity tests, changes in mean blood pressure and heart rate were analyzed using repeated-measures analysis of variance, followed by $t$-test with Bonferroni correction. $\mathrm{P}$ values $<0.05$ were considered to be significant.

\section{RESULTS}

A total of 110 patients among those who received elective ureteroscopic stone surgery under general anesthesia in our hospital between January and December 2019 satisfied the inclusion criteria. Of these, 17 patients were excluded; thus, 93 patients were included in the final analysis (control group, $\mathrm{n}=52$; chlorpheniramine group, $\mathrm{n}=41$; Fig. 1 ).

The patient characteristics and operative data were comparable between the groups (Table 1).

The intraoperative and recovery data are presented in Table 2. The incidence of EA was similar in the two groups $(21.2 \%$ [11/52] in the control group and $24.4 \%$ [10/41] in the chlorpheniramine group; odds ratio, 0.832; 95\% confidence interval: $0.3-2.2 ; \mathrm{P}=0.711)$. EA severity did not differ between groups (RSAS 3-7: control group, $\mathrm{n}=2$, 39, 8, 3, 1; chlorpheniramine group, $\mathrm{n}=4,27,5,3,2$, respectively; $\mathrm{P}=$

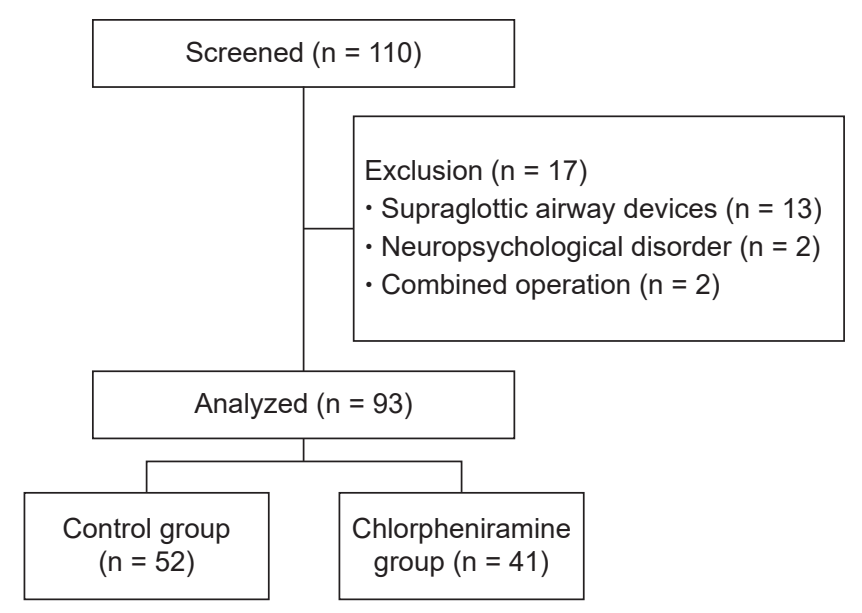

Fig. 1. Flow diagram. 
Table 1. Patient Characteristics and Operative Data

\begin{tabular}{|c|c|c|c|}
\hline Variable & Control $(n=52)$ & Chlorpheniramine $(n=41)$ & $P$ value \\
\hline Age (yr) & $49.7 \pm 10.3$ & $48.0 \pm 12.0$ & 0.485 \\
\hline $\operatorname{Sex}(M / F)$ & $34 / 18$ & $23 / 18$ & 0.361 \\
\hline Height (cm) & $162.3 \pm 9.4$ & $165.1 \pm 8.7$ & 0.147 \\
\hline Weight (kg) & $70.6 \pm 15.1$ & $70.8 \pm 14.9$ & 0.961 \\
\hline Body mass index $\left(\mathrm{kg} / \mathrm{m}^{2}\right)$ & $26.7 \pm 4.5$ & $25.8 \pm 4.4$ & 0.378 \\
\hline \multicolumn{4}{|l|}{ ASA classification } \\
\hline I/II/III & $11 / 36 / 5$ & $9 / 31 / 1$ & 0.454 \\
\hline \multicolumn{4}{|l|}{ Position of stone } \\
\hline Kidney/ureter/both & $17 / 25 / 10$ & $13 / 15 / 3$ & 0.427 \\
\hline Duration of surgery (min) & $69.7 \pm 50.9$ & $53.2 \pm 32.2$ & 0.059 \\
\hline Duration of anesthesia (min) & $97.5 \pm 52.0$ & $81.1 \pm 32.6$ & 0.093 \\
\hline Fluids (ml) & $200(150,300)$ & $200(150,300)$ & 0.715 \\
\hline \multicolumn{4}{|l|}{ Urinary catheter size (Fr) } \\
\hline $14 / 16 / 18$ & $10 / 40 / 2$ & $11 / 30 / 0$ & 0.229 \\
\hline
\end{tabular}

Values are presented as mean \pm SD, number, or median (1Q, 3Q). ASA: American Society of Anesthesiologists.
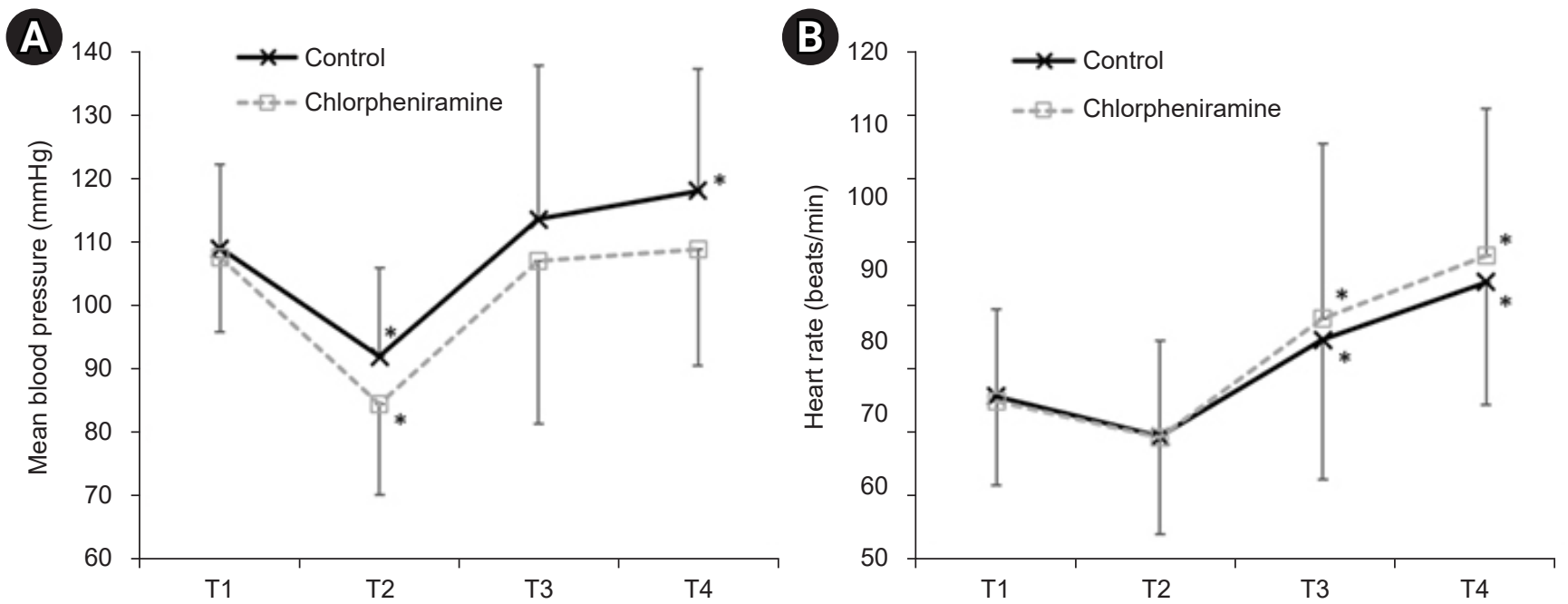

Fig. 2. Changes in mean blood pressure (A) and heart rate (B). (A) Mean blood pressure showed no intergroup difference at any of time point; $P=0.591,0.013,0.209$, and 0.021 at $\mathrm{T} 1-\mathrm{T} 4$, respectively. (B) Heart rate also showed no intergroup difference at any of time point; $\mathrm{P}=0.803,0.958,0.503$, and 0.341 at $\mathrm{T} 1-\mathrm{T} 4$, respectively. In the intergroup comparison of mean blood pressure and heart rate, $\mathrm{P}<$ 0.0125 was considered statistically significant (i.e., $0.05 / 4=0.0125$, Bonferroni correction). $\mathrm{T} 1=$ before induction of anesthesia (baseline), T2 $=$ at the end of surgery, T3 = at extubation, T4 = 5 min after extubation. Data are presented as mean \pm SD. $* P<0.05$ vs. baseline in each group (Bonferroni corrected).

0.688). Changes in mean blood pressure and heart rate were comparable between the two groups $(\mathrm{P}=0.237$ and 0.733 , respectively; Fig. 2A, B). In addition, the highest and lowest intraoperative concentrations of desflurane, time to extubation, NRS scores for postoperative pain, and numbers of patients requiring analgesics or antiemetics in the PACU were similar in the two groups (Table 2).

All adverse events are presented in Table 3; no difference was detected between groups.

\section{DISCUSSION}

In this retrospective cohort study, a single dose of chlorpheniramine administered before the induction of anesthesia was not associated with a decrease in the incidence or severity of EA in adult patients undergoing ureteroscopic stone surgery under desflurane anesthesia.

The etiology of EA is not known. EA has been reported more often in the context of the use of newer, short-acting 
Table 2. Intraoperative and Recovery Data

\begin{tabular}{|c|c|c|c|c|}
\hline Variable & Control $(n=52)$ & Chlorpheniramine $(n=41)$ & Mean difference (95\% Cl) & $\mathrm{P}$ value \\
\hline \multicolumn{5}{|l|}{ In operating room } \\
\hline \multicolumn{5}{|c|}{ Desflurane concentration, vol\% } \\
\hline Lowest concentration & $5.8(5.0,6.0)$ & $5.0(5.0,6.0)$ & $0.2(-0.2,0.6)$ & 0.361 \\
\hline Highest concentration & $6.0(5.0,6.0)$ & $6.0(5.0,6.0)$ & $0.2(-0.1,0.6)$ & 0.149 \\
\hline Time to extubation (min) & $8.0(6.3,9.0)$ & $7.3(5.5,8.6)$ & $0.4(-0.8,1.6)$ & 0.210 \\
\hline $\operatorname{RSAS}(3 / 4 / 5 / 6 / 7)^{*}$ & $2 / 39 / 8 / 2 / 1$ & $4 / 27 / 5 / 3 / 2$ & NA & 0.688 \\
\hline Emergence agitation & $11(21.2)$ & $10(24.4)$ & $-3.2(-20.6,13.4)$ & 0.711 \\
\hline NRS for pain ${ }^{\dagger}$ & $2.0(1.0,3.0)$ & $1.0(0,2.5)$ & $0.7(-0.05,1.4)$ & 0.055 \\
\hline Analgesics & $3(5.8)$ & $1(2.4)$ & $3.3(-7.5,13.4)$ & 0.628 \\
\hline Antiemetics & $2(3.8)$ & $0(0)$ & $3.8(-5.1,13.0)$ & 0.502 \\
\hline
\end{tabular}

Values are presented as median (1Q, 3Q), number, or number (\%). Time to extubation was defined as the interval between turning off the inhalation anesthetics and extubation. Emergence agitation was defined as RSAS $\geq 5$. Cl: confidential interval, RSAS: Ricker SedationAgitation Scale. ${ }^{*} 3$ = sedated, $4=$ calm and cooperative, $5=$ agitated but responding calmly to verbal instructions, $6=$ very agitated and requiring restraint, $7=$ pulling at the tracheal tube, trying to remove catheter or striking staff. ${ }^{\dagger} 0=$ no pain, $10=$ worst imaginable pain.

Table 3. Adverse Events in the Post-anesthesia Care Unit

\begin{tabular}{llcc}
\hline \multicolumn{1}{c}{ Variable } & $\begin{array}{c}\text { Control } \\
(\mathrm{n}=52)\end{array}$ & $\begin{array}{c}\text { Chlorpheniramine } \\
(\mathrm{n}=41)\end{array}$ & P value \\
\hline Sore throat & $8(15.4)$ & $11(26.8)$ & 0.174 \\
Hoarseness & $1(1.9)$ & $0(0)$ & $>0.999$ \\
Dry mouth & $2(3.8)$ & $1(2.4)$ & $>0.999$ \\
Nausea & $3(5.8)$ & $0(0)$ & 0.252 \\
Vomiting & $2(3.8)$ & $0(0)$ & 0.502 \\
Headache & $0(0)$ & $2(4.9)$ & 0.192 \\
Dizziness & $0(0)$ & $1(2.4)$ & 0.441 \\
Dyspnea & $1(1.9)$ & $0(0)$ & $>0.999$ \\
Diarrhea & $1(1.9)$ & $0(0)$ & $>0.999$ \\
\hline
\end{tabular}

Values are presented as number (\%).

halogenated compounds, such as desflurane and sevoflurane, than with the use of other inhaled anesthetics [15]. Proposed hypotheses for EA seen with desflurane use include rapid emergence with insufficient time to adjust to the strange environment, late recovery of cognitive function compared to other brain functions resulting in altered cognitive perception, increased pain sensation, and activation of the sympathetic nervous system [16].

Although the etiology of EA remains unknown, extended duration of surgery, CRBD, PONV, anticholinergics, type of surgery (e.g., otolaryngological and oral cavity surgeries), pain, and the presence of invasive devices (e.g., urinary catheter, tracheal tube, or chest tube) contributed to EA in adult patients undergoing general anesthesia [1-4]. Drugs that prevent EA include propofol, $N$-methyl-D-aspartate receptor antagonists (e.g., magnesium sulfate, ketamine, and tramadol), $\alpha_{2}$-adrenoreceptor agonists (clonidine and dex- medetomidine), and $\mu$-opioid agonists (e.g., fentanyl and remifentanil); these drugs have sedative and/or analgesic effects in common [13].

Antihistamines are among the drugs used most commonly during the perioperative period [17], and some researchers have recommended routine prophylaxis with an antihistamine to prevent life-threatening histamine-related consequences after the induction of anesthesia [18]. Depending on their impacts on the central nervous system, $\mathrm{H}_{1}$ antihistamines are classified into first-generation sedating antihistamines and second-generation antihistamines that provide less or no sedation [6].

Chlorpheniramine is a first-generation $\mathrm{H}_{1}$ receptor antagonist ( $\mathrm{H}_{1}$ antihistamine) and one of the most potent antiallergic agents in the alkylamine group; thus, it is commonly used to prevent or treat hypersensitivity and allergic reactions [8]. In addition, chlorpheniramine has sedating, antinociceptive, antiemetic, anti-inflammatory, and antimuscarinic effects $[9,19]$. These effects were expected to have a positive influence on EA, but chlorpheniramine was not associated with EA attenuation in this study. Possible explanations are as follows. First, although chlorpheniramine provides a sedative effect by penetrating the blood-brain barrier and acting on central $\mathrm{H}_{1}$ receptors, it can impair cognitive and psychomotor performance, cause problems with coordination, and, paradoxically, cause excitability and restlessness, even at therapeutic doses [6]. These effects may contribute to EA by further delaying the recovery of cognitive function after desflurane anesthesia. Second, previous studies have shown that anti-inflammatory and antimuscarinic agents (e.g., paracetamol, oxybutynin, tolterodine, glycopyr- 
rolate, and butylscopolamine) reduce CRBD [20,21]. However, in a recent study [22], chlorpheniramine decreased rescue tramadol usage to relieve postoperative CRBD, but did not reduce the incidence or severity of CRBD. The authors speculated that an $8 \mathrm{mg}$ dose of chlorpheniramine is insufficient to reduce CRBD incidence and severity because of its weak antimuscarinic effects $[7,22]$. In addition, chlorpheniramine acts on serotoninergic and cholinergic receptors, which can cause adverse effects, such as dizziness, tinnitus, anxiety, blurred vision, problems with concentration, dry mouth, and difficulty urinating [23]. These effects may have influenced the results of this study. Third, in this study, postoperative NRS scores for pain were low (medians = 1 and 2) in both groups, and only a few patients in the control group complained of PONV. These findings suggest that postoperative pain and PONV may not be important risk factors for EA in patients undergoing ureteroscopic stone surgery. Consequently, the antinociceptive and antiemetic effects of chlorpheniramine may not contribute to the attenuation of EA. On the other hand, a recent study [24] demonstrated a reduction in EA severity after functional endoscopic sinus surgery with a single preoperative dose $(5 \mathrm{mg})$ of chlorpheniramine. The discrepancy between these results may be attributed to differences in the anesthetic agents (desflurane vs. sevoflurane), types of surgery (urological vs. nasal), EA assessment tool (RSAS vs. the Richmond Agitation-Sedation Scale), and assessment period [2].

The incidence of EA in this study was lower than the $63.5 \%$ reported in patients with urinary catheters [3]. This difference may reflect the evaluation of EA only in patients undergoing ureteroscopic stone surgery, which causes less postoperative pain, in this study, whereas previous studies included patients undergoing various types of surgery known to be associated with high risks of EA, such as oral cavity, otolaryngological, and orthopedic and abdominal surgeries $[2,3]$. In contrast, the incidence of EA in our study was more than double that of $9.8 \%$ reported in patients undergoing urological surgery [4]. However, not all patients in that study had urinary catheters, and some patients had surgery under general anesthesia comprising total intravenous anesthesia and/or induced with a supraglottic airway device [4]. Total intravenous anesthesia is protective against EA [25], and supraglottic airway devices may have induced less EA compared to endotracheal tubes [26].

In a previous study, intravenous chlorpheniramine (8 mg) caused no significant hemodynamic change during anes- thesia [17]. However, EA itself can cause hemodynamic changes (e.g., hypertension and tachycardia) by increasing the sympathetic tone during emergence [13]. In this study, the mean blood pressure and heart rate during emergence did not differ between groups, supporting the lack of a significant difference in EA between groups.

The effect of the difference in anesthesia depth according to differences in inhalation anesthetic concentrations on EA is controversial $[27,28]$. In this study, desflurane concentrations were adjusted under PSI monitoring in both groups, and the highest and lowest desflurane doses during anesthesia were comparable between groups. Therefore, effects of the depth of anesthesia on EA could be excluded.

This study has some limitations. First, all patients received 1-2 $\mu \mathrm{g} / \mathrm{kg}$ fentanyl during the induction of anesthesia. In a meta-analysis of data from 3,172 children, fentanyl showed a prophylactic effect against desflurane-related EA [29]. Thus, fentanyl may have contributed to the reduction of EA in both groups in this study. Second, in this study, the duration in PACU was not included in the emergence period. Considering that all patients were urinary-catheterized and that chlorpheniramine had little effect on CRBD incidence or severity [22], it is likely that we underestimated EA incidence and severity in both groups. Third, even though the anesthesia protocols and types of surgery were uniform, individual differences in practice among anesthesiologists and surgeons may have influenced the results. Finally, this study had a retrospective design, and chlorpheniramine was not administered for EA prevention. The effects of the drugs on EA may depend on the dose and timing of administration [2]. Therefore, prospective studies are needed with controlled dosages and timing of chlorpheniramine administration.

In conclusion, a single 8-mg bolus dose of chlorpheniramine administered before anesthesia induction was not associated with a decrease in EA incidence or severity following ureteroscopic stone surgery.

\section{FUNDING}

None.

\section{CONFLICTS OF INTEREST}

No potential conflict of interest relevant to this article was reported. 


\section{DATA AVAILABILITY STATEMENT}

The datasets generated during and/or analyzed during the current study are available from the corresponding author on reasonable request.

\section{AUTHOR CONTRIBUTIONS}

Conceptualization: Tae-Yun Sung. Data curation: ChoonKyu Cho, Minhye Chang, Tae-Yun Sung. Formal analysis: Minhye Chang, Sung-Ae Cho, Tae-Yun Sung. Writing - original draft: Choon-Kyu Cho, Tae-Yun Sung. Writing - review \& editing: Choon-Kyu Cho, Tae-Yun Sung. Investigation: Minhye Chang, Tae-Yun Sung.

\section{ORCID}

Choon-Kyu Cho, https://orcid.org/0000-0001-9906-1396

Minhye Chang, https://orcid.org/0000-0002-1257-4533

Seok-Jin Lee, https://orcid.org/0000-0001-7894-8510

Sung-Ae Cho, https://orcid.org/0000-0002-1519-3787

Tae-Yun Sung, https://orcid.org/0000-0002-0714-1477

\section{REFERENCES}

1. Fields A, Huang J, Schroeder D, Sprung J, Weingarten T. Agitation in adults in the post-anaesthesia care unit after general anaesthesia. Br J Anaesth 2018; 121: 1052-8.

2. Lee SJ, Sung TY. Emergence agitation: current knowledge and unresolved questions. Korean J Anesthesiol 2020; 73: 471-85.

3. Yu D, Chai W, Sun X, Yao L. Emergence agitation in adults: risk factors in 2,000 patients. Can J Anaesth 2010; 57: 843-8.

4. Kim HC, Kim E, Jeon YT, Hwang JW, Lim YJ, Seo JH, et al. Postanaesthetic emergence agitation in adult patients after general anaesthesia for urological surgery. J Int Med Res 2015; 43: 226-35.

5. Hur M, Park SK, Yoon HK, Yoo S, Lee HC, Kim WH, et al. Comparative effectiveness of interventions for managing postoperative catheter-related bladder discomfort: a systematic review and network meta-analysis. J Anesth 2019; 33: 197-208.

6. van Schoor J. Antihistamines: a brief review. Prof Nurs Today 2012; 16: 16-21.

7. Mahdy AM, Webster NR. Histamine and antihistamines. Anaesth Intensive Care Med 2011; 12: 324-9.

8. Tzeng JI, Lin HT, Chen YW, Hung CH, Wang JJ. Chlorpheniramine produces spinal motor, proprioceptive and nociceptive blockades in rats. Eur J Pharmacol 2015; 752: 55-60.
9. Morita T, Tei Y, Shishido H, Inoue S. Chlorpheniramine maleate as an alternative to antiemetic cyclizine. J Pain Symptom Manage 2004; 27: 388-90.

10. Rose DK. Recovery room problems or problems in the PACU. Can J Anaesth 1996; 43(5 Pt 2): R116-28.

11. Laguna JJ, Archilla J, Doña I, Corominas M, Gastaminza G, Mayorga C, et al. Practical guidelines for perioperative hypersensitivity reactions. J Investig Allergol Clin Immunol 2018; 28 216-32.

12. Abdellatif AA, Kamal MM, Ishak RA. Addition of dexamethasone-chlorpheniramine mixture reduces the incidence of vomiting associated with oral ketamine premedication after pediatric dental procedures. Ain-Shams J Anaesthesiol 2016; 9: $478-84$.

13. Lee SJ, Choi SJ, In CB, Sung TY. Effects of tramadol on emergence agitation after general anesthesia for nasal surgery: a retrospective cohort study. Medicine (Baltimore) 2019; 98: e14763.

14. Riker RR, Picard JT, Fraser GL. Prospective evaluation of the Sedation-Agitation Scale for adult critically ill patients. Crit Care Med 1999; 27: 1325-9.

15. Vlajkovic GP, Sindjelic RP. Emergence delirium in children: many questions, few answers. Anesth Analg 2007; 104: 84-91.

16. Cohen IT, Hannallah RS, Hummer KA. The incidence of emergence agitation associated with desflurane anesthesia in children is reduced by fentanyl. Anesth Analg 2001; 93: 88-91.

17. Hahm TS, Kim CS, Koo MS, Shin BS, Hwang HY, Lee SM, et al. The effect of H1-receptor antagonist on hemodynamic change during anesthesia. Korean J Anesthesiol 2006; 51: 395-9.

18. Lorenz W, Duda D, Dick W, Sitter H, Doenicke A, Black A, et al. Incidence and clinical importance of perioperative histamine release: randomised study of volume loading and antihistamines after induction of anaesthesia. Trial Group Mainz/Marburg. Lancet 1994; 343: 933-40.

19. Raffa RB. Antihistamines as analgesics. J Clin Pharm Ther 2001; 26: $81-5$.

20. Ergenoglu P, Akin S, Yalcin Cok O, Eker E, Kuzgunbay B, Turunc $\mathrm{T}$, et al. Effect of intraoperative paracetamol on catheter-related bladder discomfort: a prospective, randomized, double-blind study. Curr Ther Res Clin Exp 2012; 73: 186-94.

21. Kim JA, Min JH, Lee HS, Jo HR, Je UJ, Paek JH. Effects of glycopyrrolate premedication on preventing postoperative catheter-related bladder discomfort in patients receiving ureteroscopic removal of ureter stone. Korean J Anesthesiol 2016; 69: 563-7.

22. In CB, Lee SJ, Sung TY, Cho CK, Jee YS. Effects of chlorpheniramine maleate on catheter-related bladder discomfort in pa- 
tients undergoing ureteroscopic stone removal: a randomized double-blind study. Int J Med Sci 2021; 18: 1075-81.

23. Simons FE. H1-antihistamines: more relevant than ever in the treatment of allergic disorders. J Allergy Clin Immunol 2003; 112(4 Suppl): S42-52.

24. Abdelrahman TN, Kasem AA. Role of preemptive chlorpheniramine maleate in reducing postoperative agitation after functional endoscopic sinus surgeries (FESS). Ain-Shams J Anesthesiol 2020; 12: 26

25. Chen L, Xu M, Li GY, Cai WX, Zhou JX. Incidence, risk factors and consequences of emergence agitation in adult patients after elective craniotomy for brain tumor: a prospective cohort study. PLoS One 2014; 9: e114239.

26. Keles S, Kocaturk O. Postoperative discomfort and emergence delirium in children undergoing dental rehabilitation under general anesthesia: comparison of nasal tracheal intubation and laryngeal mask airway. J Pain Res 2018; 11: 103-10.

27. Frederick HJ, Wofford K, de Lisle Dear G, Schulman SR. A randomized controlled trial to determine the effect of depth of anesthesia on emergence agitation in children. Anesth Analg 2016; 122: 1141-6.

28. Holzki J, Kretz FJ. Changing aspects of sevoflurane in paediatric anaesthesia: 1975-99. Paediatr Anaesth 1999; 9: 283-6.

29. Dahmani S, Stany I, Brasher C, Lejeune C, Bruneau B, Wood C, et al. Pharmacological prevention of sevoflurane- and desflurane-related emergence agitation in children: a meta-analysis of published studies. Br J Anaesth 2010; 104: 216-23. 